\title{
Rasmussen's Encephalitis
}

National Institute of Neurological Disorders and Stroke (NINDS)

\section{Source}

National Institute of Neurological Disorders and Stroke (NINDS). Rasmussen's

Encephalitis Information Page.

Rasmussen's encephalitis (RE) is a very rare, chronic inflammatory neurological disease that usually affects only one hemisphere (half) of the brain. It most often occurs in children under the age of 10 but can also affect adolescents and adults. It has features of an autoimmune disease in which immune system cells enter the brain and cause damage. RE is characterized by frequent and severe seizures, progressive loss of neurological functions including motor skills, speech, and eventual paralysis on one side of the body (hemiparesis), inflammation of the brain (encephalitis), and mental deterioration. Children with RE frequently enter a phase of permanent, but stable, neurological deficits after 8 to 12 months; the disease in adults and adolescents may continue to progress slowly. 